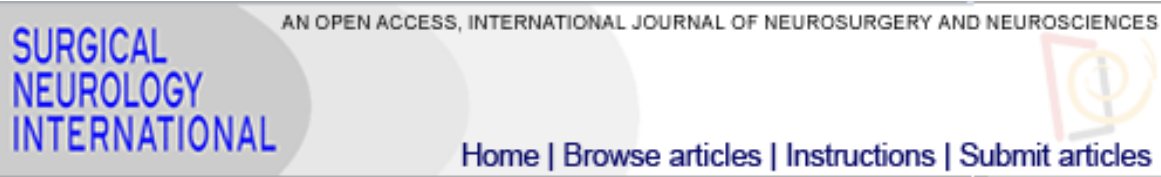

Surg Neurol Int. 2017; 8: 298.

PMCID: PMC5735431

Published online 2017 Dec 6. doi: 10.4103/sni.sni 1917

\title{
Deep brain stimulation for obsessive-compulsive disorder: Knowledge and concerns among psychiatrists, psychotherapists and patients
}

Matilda Naesström, $^{1,{ }^{*}}$ Patric Blomstedt, ${ }^{2}$ Marwan Hariz, $^{2,3}$ and $\underline{\text { Owe Bodlund }}^{1}$

${ }^{1}$ Department of Clinical Sciences/Psychiatry, Umeå University, Sweden

2 Unit of Deep Brain Stimulation, Department of Pharmacology and Clinical Neuroscience, Umeå University, Sweden

${ }^{3}$ Unit of Functional Neurosurgery, UCL Institute of Neurology, Queen Square, London, United Kingdom

Matilda Naesström: matilda.naesstrom@umu.se; Patric Blomstedt: patric.blomstedt@umu.se; Marwan Hariz: marwan.hariz@umu.se; Owe

Bodlund: owe.bodlund@umu.se

* Corresponding author

Received 2017 Jan 10; Accepted 2017 May 15.

Copyright : (c) 2017 Surgical Neurology International

This is an open access article distributed under the terms of the Creative Commons Attribution-NonCommercial-ShareAlike 3.0 License, which allows others to remix, tweak, and build upon the work non-commercially, as long as the author is credited and the new creations are licensed under the identical terms.

\section{Abstract}

\section{Background:}

Deep brain stimulation (DBS) is under investigation for severe obsessive-compulsive disorder (OCD) resistant to other therapies. The number of implants worldwide is slowly increasing. Therefore, it is of importance to explore knowledge and concerns of this novel treatment among patients and their psychiatric healthcare contacts. This information is relevant for scientific professionals working with clinical studies for DBS for this indication. Especially, for future study designs and the creation of information targeting healthcare professionals and patients. The aim of this study was to explore the knowledge and concerns toward DBS among patients with OCD, psychiatrists, and cognitive behavioral therapists.

\section{Methods:}

The study was conducted through web-based surveys for the aimed target groups -psychiatrist, patients, and cognitive behavioral therapists. The surveys contained questions regarding previous knowledge of DBS, source of knowledge, attitudes, and concerns towards the therapy.

\section{Results:}

The main source of information was from scientific sources among psychiatrists and psychotherapists. The patient's main source of information was the media. Common concerns among the groups included complications from surgery, anesthesia, stimulation side effects, and the novelty of the treatment. Specific concerns for the groups included; personality changes mentioned by patients and psychotherapists, and ethical concerns among psychiatrists.

\section{Conclusion:}

There are challenges for DBS in OCD as identified by the participants of this study; source and quality of information, efficacy, potential adverse effects, and eligibility. In all of which the current evidence base still is limited. A broad research agenda is needed for studies going forward.

Keywords: Attitude, deep brain stimulation, obsessive-compulsive disorder, patient, psychiatrist, psychotherapist 
Obsessive-compulsive disorder (OCD) is a chronic disorder affecting approximately $2 \%$ of the population. The disorder is characterized by persistent obsessive, intrusive thoughts generating anxiety, and related compulsions (tasks or "rituals") with the function of neutralizing the distress. [4] This disorder is one of the most disabling psychiatric disorders and it comes with a significant mortality. Alonso et al. suggest that 10 $27 \%$ of the patients might attempt suicide during their lifetime.[2] Further, up to $10 \%$ of patients with OCD continues to demonstrate severe therapy-refractory symptoms despite trying multiple available treatments. $[\underline{9}, \underline{10}]$

New treatments are currently under evaluation, including deep brain stimulation (DBS), which modulates brain circuits hypothesized to be implicated in OCD. DBS is an established treatment for severe therapyrefractory Parkinson's disease, dystonia, and essential tremor and is currently evaluated for a number psychiatric disorders.[19] One of the most well-studied psychiatric disorders is OCD. DBS for OCD is not yet an established therapy, but has received an FDA approval as a "humanitarian device exemption," thus advancing further forward from experimental to limited clinical use.[1] In the literature data from around 100 patients with severe OCD has been published.[21] The majority of patients were evaluated within a large number of mostly small and nonrandomized studies targeting different brain structures. However, the results have been promising with a symptom reduction typically about $50 \%$ and minor side effects. The question remains how come there are no more than about 100 patients reported to have undergone DBS for OCD in the literature, despite 17 years since the first publication, the debilitating symptoms in the severe form of the disorder, lack of therapeutic options, and promising results of DBS? Therefore, it is important to explore knowledge and concerns of DBS among patients with OCD and their potential referrers.

DBS trials for psychiatric conditions require collaboration between multidisciplinary teams highly specialized in DBS, participating patients, primary psychiatric healthcare providers, and often also involvement of other caretakers and family members. This invasive and probably life-long treatment, differs considerably from the established therapies, and was in one study ranked as the least preferred novel treatment among inpatients with OCD.[7] It is therefore of importance to gain an understanding of the level of knowledge and existing concerns among both medical staff and patients regarding this novel therapy. Such data can be of value to professional and public debate, guidelines and policymaking concerning DBS for OCD.

Few publications have been presented regarding quality of life and experiences in OCD patients after treatment with DBS.[묘,24] The authors are; however, unaware of any studies that have examined the knowledge of, and attitude towards DBS in patients with OCD not enrolled in a DBS study. The same is also true regarding their medical mental-health carers; psychiatrists and psychotherapists. Therefore, the aim of this study was to identify level of knowledge and concerns, which might be relevant for future study designs and creation of information targeting healthcare professionals and patients.

The study was conducted through a web-based survey, specifically aimed to psychiatrists, cognitive behavioral therapy (CBT) psychotherapists, and patients with OCD. Given the relatively small sample sizes, and the paucity of previous research in this area, the study was exploratory.

\section{MATERIALS AND METHODS}

Three web-based surveys were constructed for psychiatrist, therapists with experience of CBT, and OCD patients, respectively. The surveys contained questions concerning age, sex, previous knowledge of DBS, source of knowledge, attitudes, and concerns towards the therapy. The patient survey included additional questions regarding self-assessed severity of the disorder and current treatment regime. The link to the websurvey was distributed among psychiatrists in the northern region of Sweden and a national CBT psychotherapist group. For patients with OCD, a link was published at the website of the national Swedish OCD patient-support group. The data was analyzed using the statistical tool SPSS, version 22 . The project was approved by the Umeå ethics board.

\section{RESULTS AND DISCUSSION}

A total of 65 patients with OCD, 44 psychiatrists, and 52 psychotherapists answered the survey.

Characteristics of the target groups, sources of information, and concerns are presented in Tables $\underline{1}-\underline{3}$. 


\section{Patients}

In response to the question how large an impairment OCD had on their social and professional life $3.1 \%$ answered no, $15.4 \%$ minor, $24.6 \%$ moderate, $41.5 \%$ major, and $15.4 \%$ extreme impairment. Of the patients $38.5 \%$ had a combination of pharmacological therapy and psychotherapy, $35.4 \%$ only pharmacological therapy and $7.7 \%$ only psychotherapy; while $18.5 \%$ received no current treatment. $58.5 \%$ of the patients were positive to consider undergoing DBS for OCD. Males were more positive than females $(P$ value 0.05 , Pearson's Chi-squared test), but no differences were seen regarding age. The most common concerns regarding DBS are presented in Table 3.

\section{Psychiatrists}

Of the responders $57 \%$ were certified psychiatrists and $43 \%$ under specialist-training in psychiatry. $93.2 \%$ had knowledge of DBS for OCD prior to the survey. After being presented with a list of inclusion criteria for DBS (diagnosis of severe and debilitating OCD and nonresponse to trial of CBT and at least three seretonin reuptake inhibitors (SRI) trials, including with augmentation of a neuroleptic) 50\% estimated that among their patients 2-5 fulfilled these criteria, 34\% 6-10 patients fulfilling the criterion, $14 \%>10$ patients, and $2 \%$ no patients. A majority (95\%), were positive to refer patients with OCD for DBS.

\section{Cognitive behavioral therapy psychotherapists}

All responders had experiences with CBT for OCD, $46 \%$ had a degree in psychology and the remainder had additional training in CBT. After being presented with a list of inclusion and exclusion criteria for DBS in OCD 54\% estimated that their clinic had no patients that could fulfill these criteria, 38\% 2-5 patients, 4\% 610 patients, and $4 \%>10$ patients. $94 \%$ were positive to DBS for OCD.

\section{Knowledge of deep brain stimulation}

Of the OCD patients $29 \%$ had knowledge of DBS prior to the survey. The majority had obtained this information through media and there was a nonsignificant trend suggesting that patients with previous knowledge of DBS were more positive to undergoing such treatment.

It has previously been pointed out that patients educate themselves and build their hopes from uncritical media sources, which might be an issue since media cover on DBS usually is overly optimistic with minimal coverage of risks. $[\underline{29}, \underline{31}]$ Concern has also been raised for DBS gaining public popularity before a full evaluation of effectiveness and adverse-effects in psychiatric indications is undertaken. $[\underline{14}, \underline{28}, \underline{30}]$

This highlights the importance of establishing a dialogue between experts and the general public to foster a better understanding of the possibilities and limitations of DBS. Ultimately, scientists and physicians in the field need to consider the public portrayal of DBS for OCD, to ensure realistic hopes.

In comparison, the main source of information for psychiatrists and psychotherapists consisted scientific sources and colleagues. The waste majority of psychiatrists (93.1\%) and almost half of the CBT therapists (40.3\%) had previous knowledge of DBS. This could reflects the high interest for the field, which can be seen in the increasing number of publications related to DBS for psychiatric indications.[15]

Considering the novelty and invasiveness of DBS in psychiatry, a majority of psychiatrists (95\%), CBT psychotherapists (94\%), and OCD patients (58.5\%) were positive to DBS as a therapy for OCD. However, there is a risk for selection bias with primarily individuals with an interest in DBS participating in the study. That over half of the patients could consider treatment with DBS contrasts with a previous study from 2010 by Patel et al., where DBS was ranked as the least preferred investigational treatment among patients with OCD.[26] Similarly with Patel et al. we found that males were more positive towards DBS than females.

The positive attitude towards DBS among psychiatrists and CBT psychotherapists is reassuring considering the potential need for medical follow-up for future DBS OCD patients in their own community. Two commonly mentioned concerns among psychiatrist were resistance to neurosurgery among potential patients and the difficulty to identify eligible candidates. This could represent a lack of knowledge of selection criteria for OCD trials and risks, and possibilities of DBS. Strategies to communicate selection criteria for patient referral to psychiatrist and to develop clear psychiatric postsurgical follow-up strategies for the patients' primary psychiatric contacts will be needed in the future. It will further be of importance to optimize care and 
transfer of knowledge to local psychiatric health contacts for this novel patient group. Furthermore, CBT has been suggested as a promising augmentation of DBS in OCD. $[\underline{8}, \underline{20}]$ This encourages an increased involvement of CBT psychotherapists.

\section{Concerns regarding deep brain stimulation}

Complications from surgery and anesthesia was a common concern in all three groups. This is similar to Leykin et al., where participants in a DBS-trial for treatment-resistant depression correctly identified the surgery itself as the riskiest part of the study.[17] As pointed out by Lipsman et al. "Although DBS is minimally invasive neurosurgery, it is the maximally invasive psychiatric treatment available." [18] DBS in psychiatric disorders has the advantage of being able to build on 20 years of experience with DBS in movement disorders. The surgical procedure and the risk for complications do not differ greatly from its use in more well studied indications, e.g., Parkinson's disease.[5]

Side-effects of the stimulation were a main concern in the patient group. Compared to the extensive data on surgical complications, there is limited knowledge about the potential stimulation related adverse effects of DBS in psychiatric patients. Multiple different anatomical targets are under evaluation in OCD (nucleus accumbens, ventral capsule/ventral striatum, subthalamic nucleus, internal capsule, inferior thalamic peduncle, bed nucleus of stria terminalis) and they differ in probability and quality of stimulation induced side-effects.[21] Related to the issue with multiple targets under investigation is the concern from patients and CBT psychotherapists of limited effect of DBS on symptoms. Thus, further research is needed to establish the efficacy of, safety of, and eligibility for the different targets for OCD.

The possibility of DBS-induced personality changes was the second most common concern among CBT psychotherapists and the third concern in the patient group. The concept of personality is complex and it is not possible from this survey to know what the groups would define as a personality change. However, the possibility of stimulation induced changes in personality is currently one of the most discussed clinical side effect and ethical concern for DBS in psychiatric indications.[ㅁ, $25, \underline{33}]$ The majority of the authors suggest that positive changes of important elements of personality, such as mood and cognitive behavior, is an intended outcome in psychiatric patients, rather than an unwanted, coincidental side-effect. For example, a patient with OCD should be expected to display less obsessive personality traits after successful treatment. There are few reports of perceived positive and negative personality changes after treatment with DBS in patients with Parkinson's disease.[ㄱ, 16] Concerning OCD a small study by Gabriels et al. found no adverse changes in personality when using self-rated personality inventory in three patients treated with DBS in the internal capsule.[12] However, larger studies systematically looking at the effects of DBS on personality in psychiatric patients are still missing.

\section{Ethics}

The use of DBS in OCD and other psychiatric disorders faces a number of unique ethical challenges and concerns, which is not surprising considering the historical legacy of surgery in this group of patients. Hence, it is reassuring that the field appears to be aware of this issue, considering the topic is regularly discussed in the literature.[푸 $\underline{6}, \underline{11}, \underline{23}, \underline{27}, \underline{30}, \underline{32}]$

\section{Eligibility for referral to deep brain stimulation}

Considering eligibility of patients, over half of the psychotherapists reported that they had no OCD patients that would fulfill the presented inclusion criteria for DBS. This was in contrast to the psychiatrists, where only $2 \%$ estimated that they had no patients fulfilling the inclusion criteria. This discrepancy could be due to psychotherapists encountering patients motivated and eligible for CBT therapy, hence with a less severe form of the disorder, while psychiatrists might encounter a greater diversity of OCD patients, including some with severe symptoms. A study using data from a naturalistic clinical sample found that meeting the stringent criteria to qualify for DBS is rare among the general OCD population.[13] The question of the potential need for DBS in OCD and how to determine the candidates that will most likely benefit from the therapy has still to be determined. 
The interest and research in DBS in psychiatric disorders have surged the past half-decade since the first publication of Nuttin et al. in 1999, and the therapy is hoped to have the potential to relieve symptoms in some of the most disabling disorders known to humankind.[22] With the FDA approval for a "humanitarian device exemption" and the expected increase in number of worldwide implantations, DBS for OCD may be moving from experimental to limited clinical use, thus increasing the number of potential patients that will be in need of ongoing follow-up care by professionals in mental healthcare. $[1,21]$ This underlines the importance of proper education and information for potential DBS patients and their healthcare contacts. The current knowledge and experience of DBS in OCD mainly derives from small nonrandomized studies. There are still plenty of challenges identified by the participants of this study: source and quality of information, efficacy, potential adverse effects, eligibility, and the multitude of targets for DBS in psychiatry. For all of those, the current evidence base is very limited; therefore, a broad research agenda is needed for studies going forward.

\section{Limitations}

The main limitation of the study is the small sample size, which limited the type of analyses that could be performed, as well as the interpretations that could be made. In addition, there is a risk for selection bias, in the sense that the subjects who chose to participate could already have an interest in DBS. Further, it is possible that the attitude towards DBS for psychiatric disorders differ between countries, due to cultural differences and historical experiences of other forms of psychosurgery. Hence, the sample might not be representative for the targeted groups in other countries. The aim was to obtain an overview of the quality and variety of the concerns with DBS in OCD, and not necessarily to provide a representative quantification. Further studies are needed to examine the knowledge and concerns about DBS in OCD across a broader sample.

\section{Financial support and sponsorship}

Nil.

\section{Conflicts of interest}

There are no conflicts of interest.

\section{Footnotes}

http://surgicalneurologyint.com/Deep-brain-stimulation-for-obsessive-compulsive-disorder:-Knowledge-and-concernsamong-psychiatrists,-psychotherapists-and-patients/

\section{REFERENCES}

1. Humanitarian device exemption DBS for OCD. F.D.A. 2009.

https://www.accessdata.fda.gov/scripts/cdrh/cfdocs/cfhde/hde.cfm?id=H050003)

2. Alonso P, Segalas C, Real E, Pertusa A, Labad J, Jimenez-Murcia S, et al. Suicide in patients treated for obsessive-compulsive disorder: A prospective follow-up study. J Affect Disord. 2010;124:300-8.

[PubMed: 20060171]

3. Bell E, Mathieu G, Racine E. Preparing the ethical future of deep brain stimulation. Surg Neurol. 2009;72:577-86. discussion 586. [PubMed: 19608246]

4. Bjorgvinsson T, Hart J, Heffelfinger S. Obsessive-compulsive disorder: Update on assessment and treatment. J Psychiatr Pract. 2007;13:362-72. [PubMed: 18032981]

5. Blomstedt P, Hariz MI. Are complications less common in deep brain stimulation than in ablative procedures for movement disorders? Stereotact Funct Neurosurg. 2006;84:72-81. [PubMed: 16790989]

6. Cabrera LY, Evans EL, Hamilton RH. Ethics of the electrified mind: Defining issues and perspectives on the principled use of brain stimulation in medical research and clinical care. Brain Topogr. 2014;27:33-45.

[PMCID: PMC3806889] [PubMed: 23733209] 
7. Castelli L, Perozzo P, Zibetti M, Crivelli B, Morabito U, Lanotte M, et al. Chronic deep brain stimulation of the subthalamic nucleus for Parkinson's disease: Effects on cognition, mood, anxiety and personality traits. Eur Neurol. 2006;55:136-44. [PubMed: 16682797]

8. de Haan S, Rietveld E, Stokhof M, Denys D. Effects of Deep Brain Stimulation on the Lived Experience of Obsessive-Compulsive Disorder Patients: In-Depth Interviews with 18 Patients. PLoS One.

2015;10:e0135524. [PMCID: PMC4552296] [PubMed: 26312488]

9. Denys D. Pharmacotherapy of obsessive-compulsive disorder and obsessive-compulsive spectrum disorders. Psychiatr Clin N Am. 2006;29:553-584, xi.

10. Denys D, Mantione M, Figee M, van den Munckhof P, Koerselman F, Westenberg H, et al. Deep brain stimulation of the nucleus accumbens for treatment-refractory obsessive-compulsive disorder. Arch Gen Psychiatry. 2010;67:1061-8. [PubMed: 20921122]

11. Dunn LB, Holtzheimer PE, Hoop JG, Mayberg HS, Appelbaum PS. Ethical Issues in Deep Brain Stimulation Research for Treatment-Resistant Depression: Focus on Risk and Consent. AJOB Neurosci. 2011;2:29-36. [PMCID: PMC4517472] [PubMed: 26229726]

12. Gabriels L, Cosyns P, Nuttin B, Demeulemeester H, Gybels J. Deep brain stimulation for treatmentrefractory obsessive-compulsive disorder: Psychopathological and neuropsychological outcome in three cases. Acta psychiatr Scand. 2003;107:275-82. [PubMed: 12662250]

13. Garnaat SL, Greenberg BD, Sibrava NJ, Goodman WK, Mancebo MC, Eisen JL, et al. Who qualifies for deep brain stimulation for OCD. Data from a naturalistic clinical sample? J Neuropsychiatry Clin Neurosci. 2014;26:81-6. [PMCID: PMC4093791] [PubMed: 24515679]

14. Gilbert F, Ovadia D. Deep brain stimulation in the media: Over-optimistic portrayals call for a new strategy involving journalists and scientists in ethical debates. Front Integr Neurosci. 2011;5:16.

[PMCID: PMC3095813] [PubMed: 21617733]

15. Ineichen C, Christen M. Analyzing 7000 texts on deep brain stimulation: What do they tell us? Front Integr Neurosci. 2015;9:52. [PMCID: PMC4620160] [PubMed: 26578908]

16. Lewis CJ, Maier F, Horstkotter N, Zywczok A, Witt K, Eggers C, et al. Subjectively perceived personality and mood changes associated with subthalamic stimulation in patients with Parkinson's disease. Psychol Med. 2015;45:73-85. [PubMed: 25066623]

17. Leykin Y, Christopher PP, Holtzheimer PE, Appelbaum PS, Mayberg HS, Lisanby SH, et al. Participants' Perceptions of Deep Brain Stimulation Research for Treatment-Resistant Depression: Risks, Benefits, and Therapeutic Misconception. AJOB Prim Res. 2011;2:33-41. [PMCID: PMC4516276] [PubMed: 26225215]

18. Lipsman N, Bernstein M, Lozano AM. Criteria for the ethical conduct of psychiatric neurosurgery clinical trials. Neurosurg Focus. 2010;29:E9.

19. Lyons MK. Deep brain stimulation: Current and future clinical applications. Mayo Clin Proc. 2011;86:662-72. [PMCID: PMC3127561] [PubMed: 21646303]

20. Mantione M, Nieman DH, Figee M, Denys D. Cognitive-behavioural therapy augments the effects of deep brain stimulation in obsessive-compulsive disorder. Psychol Med. 2014;44:3515-22. [PubMed: 25065708]

21. Naesstrom M, Blomstedt P, Bodlund O. A systematic review of psychiatric indications for deep brain stimulation, with focus on major depressive and obsessive-compulsive disorder. Nord J Psychiatry. 2016;70:483-91. [PubMed: 27103550]

22. Nuttin B, Cosyns P, Demeulemeester H, Gybels J, Meyerson B. Electrical stimulation in anterior limbs of internal capsules in patients with obsessive-compulsive disorder. Lancet. 1999;354:1526.

23. Nuttin B, Wu H, Mayberg H, Hariz M, Gabriels L, Galert T, et al. Consensus on guidelines for stereotactic neurosurgery for psychiatric disorders. J Neurol Neurosurg Psychiatry. 2014;85:1003-8. [PMCID: PMC4145431] [PubMed: 24444853] 
24. Ooms P, Mantione M, Figee M, Schuurman PR, van den Munckhof P, Denys D. Deep brain stimulation for obsessive-compulsive disorders: Long-term analysis of quality of life. J Neurol Neurosurg Psychiatry. 2014;85:153-8. [PubMed: 23715912]

25. Pacholczyk A. DBS Makes You Feel Good!-Why Some of the Ethical Objections to the Use of DBS for Neuropsychiatric Disorders and Enhancement are Not Convincing. Front Integr Neurosci. 2011;5:14.

[PMCID: PMC3090675] [PubMed: 21577266]

26. Patel SR, Simpson HB. Patient preferences for obsessive-compulsive disorder treatment. J Clin Psychiatry. 2010;71:1434-9. [PMCID: PMC3109740] [PubMed: 21114948]

27. Rabins P, Appleby BS, Brandt J, DeLong MR, Dunn LB, Gabriels L, et al. Scientific and ethical issues related to deep brain stimulation for disorders of mood, behavior, and thought. Arch Gen Psychiatry. 2009;66:931-7. [PMCID: PMC2753479] [PubMed: 19736349]

28. Racine E, Waldman S, Palmour N, Risse D, Illes J. "Currents of hope": Neurostimulation techniques in U.S. and UK print media. Camb Q Healthc Ethics. 2007;16:312-6. [PubMed: 17695623]

29. Racine E, Waldman S, Rosenberg J, Illes J. Contemporary neuroscience in the media. Soc Sci Med. 2010;71:725-33. [PMCID: PMC2925284] [PubMed: 20609506]

30. Schlaepfer TE, Lisanby SH, Pallanti S. Separating hope from hype: Some ethical implications of the development of deep brain stimulation in psychiatric research and treatment. CNS Spectr. 2010;15:285-7. [PubMed: 20448518]

31. Schneiderman LJ. The perils of hope. Camb Q Healthc Ethics. 2005;14:235-9. [PubMed: 15887817]

32. Synofzik M, Schlaepfer TE. Electrodes in the brain--ethical criteria for research and treatment with deep brain stimulation for neuropsychiatric disorders. Brain Stimul. 2011;4:7-16. [PubMed: 21255750]

33. Synofzik M, Schlaepfer TE. Stimulating personality: Ethical criteria for deep brain stimulation in psychiatric patients and for enhancement purposes. Biotechnol J. 2008;3:1511-20. [PubMed: 19072907]

Figures and Tables

Table 1

\begin{tabular}{lccc}
\hline & OCD patients & Psychiatrist & CBT psychotherapists \\
\hline Total number & 65 & 44 & 52 \\
Males & 26 & 26 & 10 \\
Females & 39 & 18 & 42 \\
Mean age & 35 & 49 & 53 \\
\hline
\end{tabular}

OCD: Obsessive-compulsive disorder, CBT: Cognitive behavioral therapy

Group characteristics

Table 2 


\begin{tabular}{llc}
\hline Patients & Media (internet, newspaper, television) & $94.7 \%$ \\
$n=19 / 65$ & Physician & $15.7 \%$ \\
& Patient support groups & $10.5 \%$ \\
& Scientifical sources & $0 \%$ \\
Psychiatrists & Scientific sources & $78 \%$ \\
$n=41 / 44$ & Colleagues & $58.5 \%$ \\
& Media (internet, newspaper, television) & $14.6 \%$ \\
& Patients & $9.7 \%$ \\
CBT psychotherapists & Scientific sources & $61.9 \%$ \\
$n=21 / 52$ & Colleagues & $57.1 \%$ \\
& Media (internet, newspaper, television) & $28.5 \%$ \\
& Patients & $3.8 \%$ \\
\hline
\end{tabular}

OCD: Obsessive-compulsive disorder, CBT: Cognitive behavioral therapy

Previous knowledge and sources of information

Table 3

\begin{tabular}{llc}
\hline Patients & Adverse effects of stimulation & 40 \\
$n=65$ & Possibility of lack of effect on OCD symptoms & 39 \\
& Complications of surgery or anesthesia & 37 \\
& Change in personality & 37 \\
& Cosmetic concerns & 15 \\
& The need for hospitalization during surgery & 13 \\
& Limitations in daily living & 11 \\
& Worsening of OCD symptoms & 2 \\
Psychiatrists & Resistance from patients to undergo & 28 \\
& neurosurgery & \\
$n=44$ & Complications of surgery or anesthesia & 24 \\
& Difficulty identifying eligible patients & 23 \\
& Ethical concerns with neurosurgery & 13 \\
& Adverse effects of stimulation & 8 \\
& Lack of evidence & 4 \\
& Complications of surgery or anesthesia & 33 \\
& Change in personality & 20 \\
$n=52$ & Ethical concerns with neurosurgery & 18 \\
& Possibility of lack of effect on OCD symptoms & 18 \\
& Widening of inclusion criteria/not & 3 \\
& establishing that all other therapy options & \\
& have been exhausted & 3 \\
\hline & Cosmetic concerns & \\
& & \\
& &
\end{tabular}

OCD: Obsessive-compulsive disorder, CBT: Cognitive behavioral therapy, DBS: Deep brain stimulation

Concerns regarding DBS-treatment ranked in order of frequency

Articles from Surgical Neurology International are provided here courtesy of Wolters Kluwer -- Medknow 